\title{
The age-related performance decline in marathon cross-country skiing - the Engadin Ski Marathon
}

\author{
Nikolaidis, Pantelis Theodoros ; Knechtle, Beat
}

\begin{abstract}
Demographic and performance data from 197,825 athletes competing in "Engadin Ski Marathon" between 1998 and 2016 were analysed. When all finishers per age group were considered, there was no gender difference in time (2:59:00 in women versus 2:59:09 h:min:s in men; $\mathrm{P}=0.914, \eta<0.001)$ and the main effect of age group on time was trivial $(\mathrm{P}<0.001, \eta=0.007)$. When the top 10 finishers per age group were considered, men were faster than women (1:27:32 versus 1:34:19 h:min:s, respectively; $\mathrm{P}<0.001, \eta=0.373)$, there was a large effect of age group on time $(P<0.001, \eta=0.590)$ and the gender difference was larger in the older than in the younger age groups $(\mathrm{P}<0.001, \eta=0.534)$. The age of peak performance for all finishers by 1-year interval age group was 40.3 and 39.6 years in all women and men, respectively. The top 10 finishers by 1-year interval age group achieved their peak performance in the age of 38.4 and 42.2 years in women and men, respectively. The age of peak performance was older and the age-related performance decline occurred earlier in marathon cross-country skiing, compared to road-based marathon running.
\end{abstract}

DOI: https://doi.org/10.1080/02640414.2017.1325965

Posted at the Zurich Open Repository and Archive, University of Zurich

ZORA URL: https://doi.org/10.5167/uzh-152628

Journal Article

Accepted Version

Originally published at:

Nikolaidis, Pantelis Theodoros; Knechtle, Beat (2018). The age-related performance decline in marathon crosscountry skiing - the Engadin Ski Marathon. Journal of Sports Sciences, 36(6):599-604.

DOI: https://doi.org/10.1080/02640414.2017.1325965 


\section{The age-related performance decline in marathon cross- country skiing - the Engadin Ski Marathon}

\section{Running head: Age and performance in cross-country skiing}

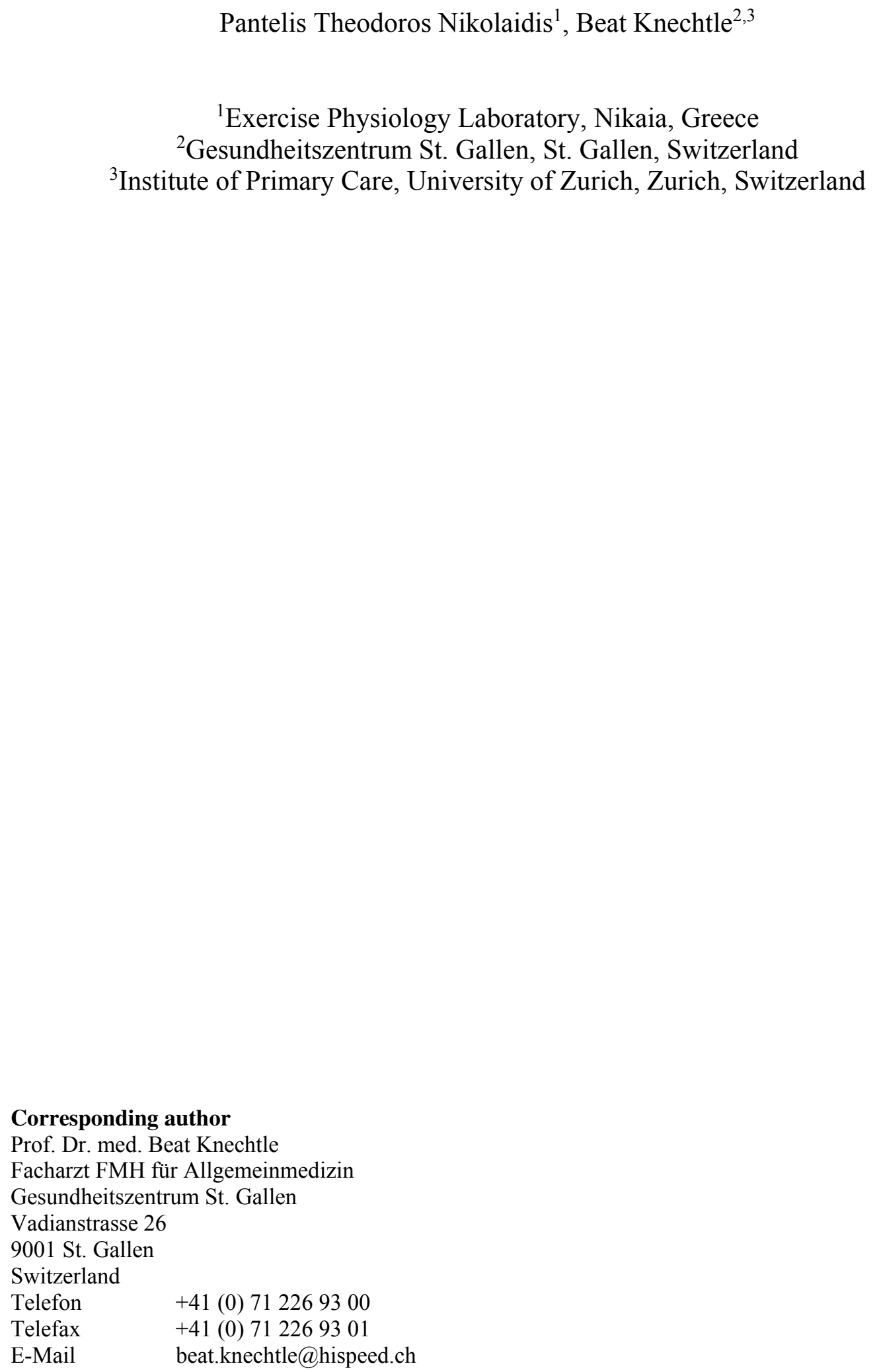

${ }^{1}$ Exercise Physiology Laboratory, Nikaia, Greece ${ }^{2}$ Gesundheitszentrum St. Gallen, St. Gallen, Switzerland ${ }^{3}$ Institute of Primary Care, University of Zurich, Zurich, Switzerland 


\section{Abstract}

The aim of the present study was to determine the age-related performance decline in the 'Engadin Ski Marathon', a cross-country race covering the marathon distance of 42.2km. Demographic and performance data from 197,825 athletes competing in this race between 1998 and 2016 were analysed. During these 17 years, $\sim 5$ times more men finished than women. When all finishers per age group were considered, there was no gender difference in race time (2:59:00 in women versus 2:59:09 h:min:s in men; $\left.p=0.914, \eta^{2}<0.001\right)$ and the main effect of age group on race time was trivial $\left(p<0.001, \eta^{2}=0.007\right)$. When the top 10 finishers per age group were considered, men were faster than women $(1: 27: 32$ versus $1: 34: 19$ h:min:s, respectively; $p<0.001$, $\left.\eta^{2}=0.373\right)$, there was a large effect of age group on race time $\left(p<0.001, \eta^{2}=0.590\right)$ and the gender difference was larger in the older than in the younger age groups $(p<0.001$, $\left.\eta^{2}=0.534\right)$. The age of peak performance for all finishers by 1 -year interval age group was 40.3 and 39.6 years in all women and men, respectively. The top 10 finishers by 1-year interval age group achieved their peak performance in the age of 38.4 and 42.2 years in women and men, respectively. In summary, the age of peak performance was older and the age-related performance decline occurred earlier in marathon crosscountry skiing compared to road-based marathon running.

Key words: athlete, female, male, winter sport 


\section{Introduction}

It is well known that athletic performance decreases with increasing age (Gava, Kern, \& Carraro, 2015; Tanaka \& Seals, 2008). The best endurance performances are often achieved at the age of $\sim 35$ years with a small and linear decline until the age of $\sim 50$ 60 years and a more pronounced decline after that age (Tanaka \& Seals, 2008).

The age-related performance decline is also dependent upon the mode of physical exercise. For example, physical performances involving mostly upper limbs (e.g. shot put, javelin throw) show a higher rate of decline ( $1.4 \%$ per year) compared to performances where the lower limbs are mostly involved (e.g. long jump 1.1\%, track events $0.6-0.7 \%$ per year) (Gava et al., 2015). Most importantly, the performance decline with ageing is related to the duration of an event, as it has been observed that sprint or power sports show higher rates of decline than endurance sports with ageing (Allen \& Hopkins, 2015).

Apart from the mode of physical performance, there is also an influence of gender on the age-related performance decline. For example, in running there is a difference in the age-related decline by both distance and gender (Zingg, Rüst, Rosemann, Lepers, \& Knechtle, 2014). In track and field athletes, differences were reported in the rates of percentage decline in running events among various distances, and significant differences between women and men were found in a study, where the decline with increasing age was greater for women and for longer endurance running events (Baker, Tang, \& Turner, 2003).

In running, the age-related performance decline has been well-investigated in female and male marathoners. Leyk et al. (2007) reported that a significant age-related 
decline in marathon running performance did not occur before the age of $\sim 50$ years. Indeed, they showed that female and male marathoners can achieve a similar race time between the ages of 20 and 50 years. A more recent study investigating female and male master marathoners competing in the 'New York City Marathon' between 1980 and 2009 showed that male ( $\geq 65$ years) and female ( $\geq 45$ years) master runners may not have reached their limits in marathon running performance (Lepers \& Cattagni, 2012).

These findings for the age-related performance decline in marathon running are most probably due to the fact that master athletes have significantly improved their performance over years (Ahmadyar, Rosemann, Rüst, \& Knechtle, 2016) whereas the performance of younger athletes has remained the same (Akkari, Machin, \& Tanaka, 2015). In running and swimming, the magnitude of improvements over four decades was greater in athletes in older age groups gradually closing the gap in athletic performance between younger and older athletes (Akkari et al., 2015). Recently, it has been shown that even athletes older than 100 years are able to achieve outstanding endurance performances (Lepers, Stapley, \& Cattagni, 2016).

Although we know the age-related performance decline for shorter (Akkari et al., 2015; Gava et al., 2015) and longer running distances such as the marathon distance (Leyk et al., 2007), we have no knowledge about the age-related performance decline in cross-country skiing, and especially on the marathon distance in cross-country skiing. Cross-country skiing is a very popular sport, especially in Scandinavian and Central European countries. Consequently, knowledge about the age-related trends in performance might be of great importance for athletes' coaches and sport scientists. Sport scientists might use this knowledge to better understand the variation of age- 

related changes among different modes of exercise, whereas coaches might develop gender- and age-tailored training goals and programs.

127 Therefore, the aim of the current study was to determine the age-related performance 128 decline in marathon cross-country skiing in the 'Engadin Ski Marathon', a cross129 country race covering the distance of a marathon $(42.195 \mathrm{~km})$. Based upon findings

130 for road-based marathon running following Leyk et al. (2007) showing that a

131 significant age-related decline in marathon running performance did not occur before

132 the age of $\sim 50$ years, we hypothesized that the age-related performance decline in 133 marathon cross-country skiing would not occur before the age of 50 years for both 134 women and men. 
149

150

151

152

153

154

155

156

157

158

159

160

161

162

163

164

165

166

167

172

\section{Methods}

\section{Ethical approval}

All procedures used in the study were approved by the Institutional Review Board of Kanton St. Gallen, Switzerland with a waiver of the requirement for informed consent of the participants given the fact that the study involved the analysis of publicly available data.

\section{The race}

All athletes who finished the 'Engadin Ski Marathon' between 1998 and 2016 were considered. Data with name, age and gender of the athletes, i.e. 34,833 women and 162,992 men, were obtained from the publicly available race website of the 'Engadin Skin Marathon' at www.engadin-skimarathon.ch.

The 'Engadin Ski Marathon' is an annually held cross-country ski race taking place on the second Sunday of March in the upper Engadin valley in Switzerland, Europe, between Maloja and S-chanf. The race started in 1969 and is one of the major crosscountry skiing events in the Swiss Alps. Each year, between 11,000 and 13,000 skiers participate in the race. Since 1998, the total distance covered is $42 \mathrm{~km}$. In that year, the race was extended by $2 \mathrm{~km}$ to match the distance of a full running marathon and the track was changed slightly resulting in a more difficult topography and longer race times. While it is a freestyle race, there are separate tracks for skiers practicing classic style for all but the narrowest parts of the race. Participation is open to anyone from the age of 16 years and no license is required to enter the event. The start of the race takes place in Maloja at the Maloja Palace Hotel with an elevation of 1,820 meters above sea level and the finish at an elevation of 1,670 meters above sea level. 
173

174

175

176

177

\section{Statistical analysis}

All statistical analyses were performed using the statistical package IBM SPSS v.20.0 (SPSS, Chicago, USA). The figures were created using the software GraphPad Prism v. 7.0 (GraphPad Software, San Diego, USA). Data were presented as mean \pm standard deviation. We examined the association of gender and age group, i.e. whether the distribution of gender varied by age group, using chi-square $\left(\chi^{2}\right)$ and Cramer's phi $(\varphi c)$ to evaluate the magnitude of association. We used two approaches to classify the participants into age groups; (a) 5-year age groups from $<20$ to $75-79$ years and (b) 1year age groups from 17 to 92 years. Furthermore, in each approach we examined both the top 10 and all skiers. The men-to-women ratio was calculated for the whole sample and for each age group. A two-way ANOVA examined the main effects of gender and age group, and the gender $\times$ age group interaction on race time, followed by a Bonferroni post-hoc analysis. The magnitude of differences in the ANOVA was evaluated using eta-squared $\left(\eta^{2}\right)$ as trivial $\left(\eta^{2}<0.01\right)$, small $\left(0.01 \leq \eta^{2}<0.06\right)$, moderate $\left(0.06 \leq \eta^{2}<0.14\right)$ and large $\left(\eta^{2} \geq 0.14\right)($ Cohen, 1988). The above mentioned ANOVA was run twice, one considering all finishers and one considering only the top 10 skiers. We used a mixed-effects regression model to examine differences in race time by gender and age group (Tabachnick \& Fidell, 2013). In the model, finisher was inserted as random variable, and gender and age group as fixed variables. In addition, we examined interaction effects between these fixed variables. Akaike information criterion (AIC) was used to select the final model. The alpha level was set at 0.05. 
197

198

199

200

201

202

203

204

205

206

207

208

209

210

211

212

213

\section{Results}

Full data with gender, age and race time from a total of 197,826 female and male athletes were available. In one athlete an extreme value was found and this athlete was deleted, leaving 197,825 athletes for data analysis.

\section{Gender $\times$ age group chi square}

The overall men-to-women ratio was 4.7. However, a gender $\times$ age group association was observed $\left(\chi^{2}=6130.13, p<0.001\right.$, Cramer's $\left.\varphi=0.176\right)$, where the men-to-women ratio was ranged from 2.4 (20-24 years age group) to 130.5 (80-84 years age group) (Figure 1). A trend was shown where the men-to-women ratio was higher in the older than in the younger age groups. In addition, the distribution of women and men in age groups followed a different pattern with most women in the age group 30-34 years whereas most men were in age group 40-44 years.

\section{Gender × age group ANOVA}

When all finishers were considered, no main effect of gender on race time was observed $\left(p=0.914, \eta^{2}<0.001\right)$, where the scores were 2:59:00 and 2:59:09 h:min:s in men and women, respectively (Figure 2). A trivial main effect of age group on race time was observed $\left(p<0.001, \eta^{2}=0.007\right)$ with the age group $40-44$ years the fastest and the oldest age group the slowest (Figure 3 ). No gender $\times$ age group interaction on race time was observed $\left(p=0.773, \eta^{2}<0.001\right)$. The findings of the mixed-effects regression analysis are presented in Table 1.

When the top 10 finishers were considered for each age group, a large main effect of gender on race time was observed $\left(p<0.001, \eta^{2}=0.373\right)$ with men significantly faster than women (1:27:32 and 1:34:19 h:min:s, respectively). Also, a large main effect of 
age group on race time was shown $\left(\mathrm{p}<0.001, \eta^{2}=0.590\right)$ with the oldest group the

221 slowest. A large gender $\times$ age group interaction on race time was observed $(p<0.001$,

$\left.222 \eta^{2}=0.534\right)$ with men significantly faster than women in most age groups and the

223 gender difference increased with age. The results of the mixed-effects regression

224 analysis are presented in Table 2.

\section{The age of peak performance}

226 The age of peak performance was 40.3 and 39.6 years in all women and men,

227 respectively, when they were examined in 1-year intervals (Figure 4). The top 10

228 finishers by 1-year interval age group achieved their peak performance at the age of

$229 \quad 38.4$ and 42.2 years in women and men, respectively.

230 When 5-year intervals were considered, the age group of peak performance was 40-44

231 years in both all women and men, but it was 40-44 years in women and 55-59 years in men when the top 10 finishers were considered. 


\section{Discussion}

This study determined the age-related performance decline in marathon cross-country skiing. It was hypothesized that this age-related decline in performance would not occur before the age of 50 years in contrast to previous findings in marathon running. The main findings of the present study were that $(i)$ the number of men finishers was $\sim 5$ times more than women and the men-to-women ratio was higher in the older than in the younger age groups; (ii) when all finishers in each age group were considered, there was no gender difference in race time and the effect of age group on race time was trivial; (iii) when the top 10 finishers in each age group were considered, men were faster than women and there was a large effect of age group on race time and the gender difference increased with age; and (iv) the age of peak performance was $\sim 40$ years for both women and men.

\section{Gender difference in performance}

A potential explanation for the difference in the fastest women and men cross-country skiers could be the fact that most women were in the age group 30-34 years and most men in the age group 40-44 years. Furthermore, the men-to-women ratio was higher in the older cross-country skiers than in the younger age groups indicating that relatively more men competed in the older age groups compared to women. Also, the men-to-women ratio was $\sim 5: 1$ in these athletes whereas in marathon running, the men-to-women ratio decreased in the 'New York City Marathon' from 5.6:1 in 1983 to 2.5:1 in 1999 indicating an increase in female athletes (Jokl, Sethi, \& Cooper, 2004). 
A major finding was that the age-related performance declined in these marathon cross-country skiers after the age of 40-44 years when all women and men were considered. However, when the 10 top skiers per age group were considered, the decline started after the age of 40-44 years in women and 50-59 years in men. This finding is in contrast to marathon running where the age-related decline in performance did not occur before the age of 50 years for both women and men (Leyk et al., 2007).

\section{Older age of peak performance compared to marathon running}

Another important finding was that the age of peak marathon cross-country performance was at the age of $\sim 40$ years for both women and men. In marathon running, the age of peak performance is at $\sim 30-35$ years, depending upon the sample and the method of analysis (Hunter, Stevens, Magennis, Skelton, \& Fauth, 2011; Lara, Salinero, \& Del Coso, 2014; Lehto, 2016; Nikolaidis, Onywera, \& Knechtle, 2016).

With regards to the general physiological functions of humans, the age of performance peak was 26 years in a study that examined the development of sport and chess performances across the lifetime (Berthelot et al., 2012).

The difference of $\sim 10$ years between marathon cross-country skiing and marathon running might be explained by differences in analyses, performance level and mode of movement. Cross-country skiing performance relies on factors limiting endurance such as maximal oxygen uptake, anaerobic threshold and exercise economy (Tanaka \& Seals, 2008) as in other exercise modes including running, cycling and swimming. On the other hand, in addition to these physiological factors, cross-country skiers need to interact efficiently with their equipment as skiing is a sport that relies more in 
technique and recruits the upper and lower body, which might explain why they maintain peak performance in older age than in other exercise modes such as marathon running.

A factor explaining the peak performance in older age in cross-country skiing compared to marathon runners could be fact that studies investigating age of peak marathon running performance have used data from flat city marathons at close to sea level (Hunter et al., 2011; Lara et al., 2014; Lehto, 2016; Nikolaidis et al., 2016) while the cross-country skiing marathon examined in the current study was held in hilly Alps and at altitude. A recent study investigating age of peak marathon running performance comparing flat city marathons and a mountain marathon showed significant differences in the age of peak marathon performance between mountain and city marathon running (Knechtle, Nikolaidis, Zingg, Rosemann, \& Rüst, 2017). In that study, the age of the top 10 women and men was older in the mountain marathon compared to the city marathons when the fastest runners were considered in 1-year age-intervals, but when all finishers were considered in 1-year age-intervals, the age of the fastest women and men was younger in the mountain marathon compared to the city marathons. An explanation of a younger age of peak performance in exercise in mountain compared to flat might be the additional muscle work and muscle power demands during exercise in mountain. Considering that the age of the peak of muscle power performance is younger than that of endurance, it is deduced that an the age of peak performance in an exercise demanding more muscle power would be younger than an exercise demanding more endurance capacity. 
317 A limitation of the present study was that since every cross-country skiing marathon possesses its own unique characteristics in terms of number participants, environmental conditions, duration and topography, the current findings should be generalized with caution. Although the findings were limited to a single skiing marathon race, they were focused on a major skiing event in Alps with large international participation. Future studies might investigate other races of the same or

323 similar distance and environmental conditions. Moreover, the large number of

324 finishers covering the adult life span was a major strength of the present study that

325 allowed for the robust examinations of differences among age groups. The genderand age-related differences observed add theoretical knowledge that may be useful for sport scientists studying the variation of performance decline with aging in different exercise modes. In addition, coaches and fitness trainers working with cross-country skiers may benefit from the findings by setting gender- and age-tailored goals and developing optimal training programs. For instance, considering that there was no gender difference in performance when all finishers were analysed, recreational athletes should be advised independent of their gender. However, since elite men were faster than women, this should be taken into account when training more competitive athletes. 
340 In marathon cross-country skiers competing in the 'Engadin Ski Marathon' between 3411998 and 2016, the age of peak performance was 40.3 and 39.6 years in all women

342 and men, when they were examined in 1-year intervals. The top 10 finishers examined 343 by 1-year intervals achieved their peak performance at the age of 38.4 and 42.2 years 344 in women and men, respectively. When the age-related performance decline was 345 considered in 5-year intervals, the age group of peak performance was 40-44 years in 346 women and men when all finishers were considered, but it was 40-44 years in women 347 and 55-59 years in men when the top 10 finishers only were considered. Compared to 348 existing findings for road-based running marathon, the age of peak performance was 349 higher and the age-related performance decline occurred earlier in marathon cross350 country skiing. 


\section{References}

Ahmadyar, B., Rosemann, T., Rüst, C.A., Knechtle, B. (2016). Improved race times in marathoners older than 75 years in the last 25 years in the World's largest marathons. Chin J Physiol, 59, 139-147. doi: 10.4077/CJP.2016.BAE382.

Akkari, A., Machin, D., \& Tanaka, H. (2015). Greater progression of athletic performance in older Masters athletes. Age Ageing, 44, 683-686. doi: 10.1093/ageing/afv023

Allen, S.V., \& Hopkins, W.G. (2015). Age of peak competitive performance of elite athletes: a systematic review. Sports Med, 45, 1431-1441. doi: 10.1007/s40279-0150354-3

Baker, A.B., Tang, Y.Q., \& Turner, M.J. (2003). Percentage decline in masters superathlete track and field performance with aging. Exp Aging Res, 29, 47-65.

Berthelot, G., Len, S., Hellard, P., Tafflet, M., Guillaume, M., Vollmer, J.-C., Gager, B., Quinquis, L., Marc, A., Toussaint, J.-F. Exponential growth combined with exponential decline explains lifetime performance evolution in individual and human species. Age, 34, 1001-9. doi: 10.1007/s11357-011-9274-9

Cohen, J. 1988. Statistical power analysis for the behavioral sciences (2nd ed.). Hillsdale, NJ: Lawrence Erlbaum Associates.

Gava, P., Kern, H., \& Carraro, U. (2015). Age-associated power decline from running, jumping, and throwing male masters world records. Exp Aging Res, 41, 115 135. doi: 10.1080/0361073X.2015.1001648

Hunter, S.K., Stevens, A.A., Magennis, K., Skelton, K.W., \& Fauth, M. (2011). Is there a sex difference in the age of elite marathon runners? Med Sci Sports Exerc, 43, 656-664. doi:10.1249/MSS.0b013e3181fb4e00

Jokl, P., Sethi, P.M., \& Cooper, A.J. (2004). Master's performance in the New York City Marathon 1983-1999. Br J Sports Med, 38, 408-412.

Knechtle, B., Nikolaidis, P.T., Zingg, M.A., Rosemann, T., \& Rüst CA. (2017). Differences in age of peak marathon performance between mountain and city marathon running - The 'Jungfrau Marathon' in Switzerland. Chin J Physiol, 60, 1122. doi: 10.4077/CJP.2017.BAE400

Lara, B., Salinero, J.J., \& Del Coso, J. (2014). The relationship between age and running time in elite marathoners is U-shaped. Age (Dordr), 36, 1003-1008. doi:10.1007/s11357-013-9614-z

Lehto, N. (2016). Effects of age on marathon finishing time among male amateur runners in Stockholm Marathon 1979-2014. J Sport Health Sci, 5, 349-354

Lepers, R., \& Cattagni, T. (2012) Do older athletes reach limits in their performance during marathon running? Age (Dordr), 34, 773-781. doi:10.1007/s11357-011-9271-z 
Lepers, R., Stapley, P.J., \& Cattagni, T. (2016). Centenarian athletes: Examples of ultimate human performance? Age Ageing, 45, 732-736. doi:10.1093/ageing/afw111

Leyk, D., Erley, O., Ridder, D., Leurs, M., Rüther, T., Wunderlich, M., Sievert, A., Baum, K., \& Essfeld, D. (2007). Age-related changes in marathon and halfmarathonperformances. Int J Sports Med, 28, 513-517.

Nikolaidis, P.T., Onywera, V.O., \& Knechtle, B. (2017). Running Performance, Nationality, Sex and Age in 10km, Half-marathon, Marathon and 100km Ultramarathon IAAF 1999-2015. J Strength Cond Res. Epub ahead of print, doi:10.1519/JSC.0000000000001687

Tabachnick, B.G., \& Fidell, L.S. (2013). Using multivariate statistics. New Jersey, USA, Pearson, 6th edition.

Tanaka, H., \& Seals, D.R. (2008). Endurance exercise performance in Masters athletes: age-associated changes and underlying physiological mechanisms. $J$ Physiol, 586, 55-63. doi:10.1113/jphysiol.2007.141879

Zingg, M.A., Rüst, C. A., Rosemann, T., Lepers, R., \& Knechtle, B. (2014). Runners in their forties dominate ultra-marathons from 50 to 3,100 miles. Clinics, 69, 203-211. 10.6061/clinics/2014(03)11

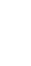


448

Table 1 Results of the mixed-effects regression analysis for race time considering all finishers in each 5-year age group

\begin{tabular}{lccc}
\hline Parameter & Estimate & SEE & P value \\
\hline Intercept & 10379.78 & 20.43 & $<0.001$ \\
Group & 57.16 & 2.92 & $<0.001$ \\
[Gender=Women] & 334.69 & 45.71 & $<0.001$ \\
[Gender=Men] & $0^{\mathrm{a}}$ & 0 &. \\
[Gender=Women] $\times$ Age group & -60.14 & 7.66 & $<0.001$ \\
[Gender=Men] $\times$ Age group & $0^{\mathrm{a}}$ & 0 &. \\
\hline
\end{tabular}

${ }^{a}$ This parameter is set to zero because it is redundant.

$\mathrm{SEE}=$ standard error of estimate. 
491

492

493

494

495

496

497

498

499

500

501

502

503

504

505

506

507

508

509

510

511

512

Table 2 Results of the mixed-effects regression analysis for race time considering the top 10 finishers in each 5-year age group

\begin{tabular}{lccc}
\hline Parameter & Estimate & SEE & P value \\
\hline Intercept & 4246.49 & 254.81 & $<0.001$ \\
Group & 262.57 & 28.02 & $<0.001$ \\
[Gender=Women] & 371.16 & 375.56 & 0.324 \\
[Gender=Men] & $0^{\mathrm{a}}$ & 0 &. \\
[Gender=Women] $\times$ Age group & -9.15 & 44.65 & 0.838 \\
[Gender =Men] $\times$ Age group & $0^{\mathrm{a}}$ & 0 &. \\
\hline
\end{tabular}

${ }^{a}$ This parameter is set to zero because it is redundant.

$\mathrm{SEE}=$ standard error of estimate.

. 
$513 \quad$ List of figures

514

515 Figure 1 Distribution of finishers by gender and age group

516

517 Figure 2 Gender difference in race time of all and top 10 finishers.

518

$*=$ significant gender difference at $\mathrm{p} \leq 0.004$.

519

520 Figure 3 Race times of all and top 10 finishers in 5-year intervals

521

522 Figure 4 Race times of all and top 10 finishers in 1-year intervals

523

524

525

526

527

528

529

530

531

532

533

534

535

536

537

538

539 
Figure 1

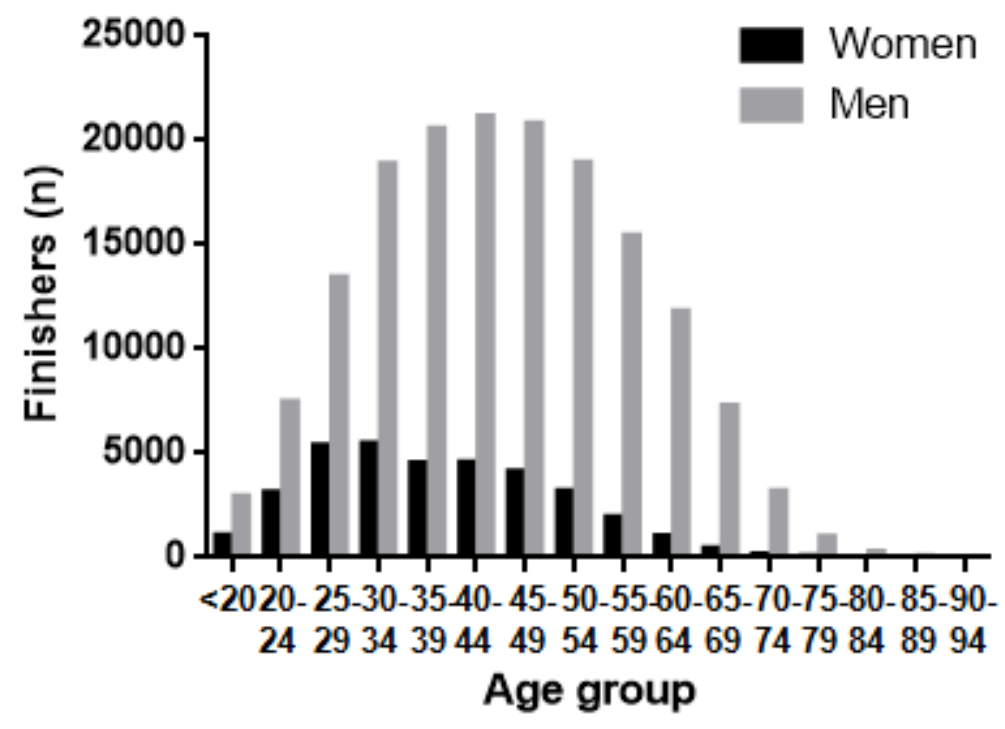

542

543

544

545

546

547

548

549

550

551

552

553

554

555

556

557

558

559 
Figure 2

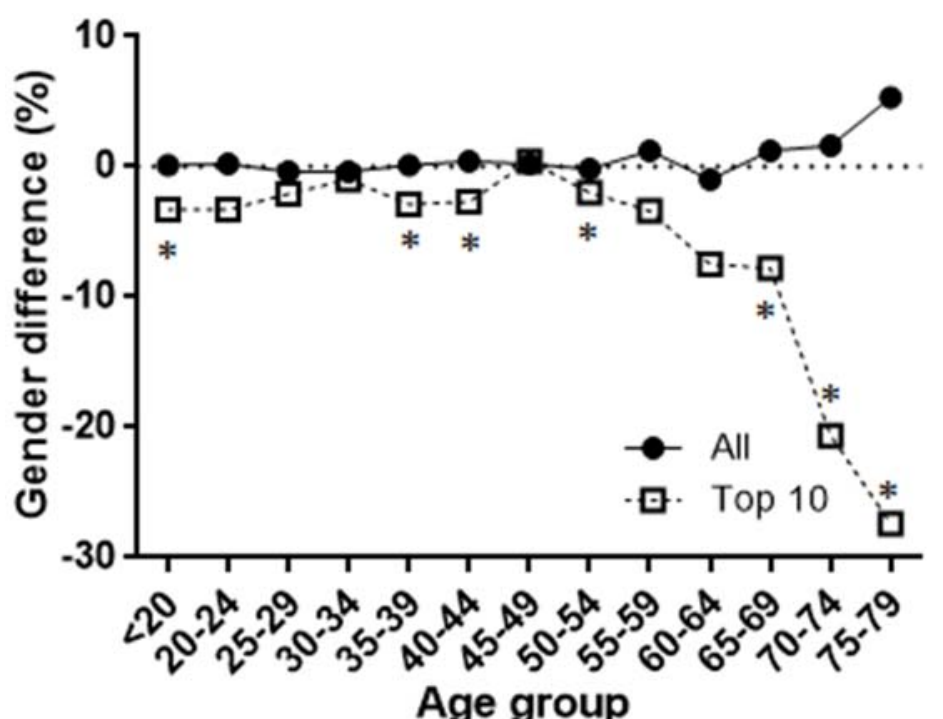

561

Age group

562

563

564

565

566

567

568

569

570

571

572

573

574

575

576

577

578 
Figure 3

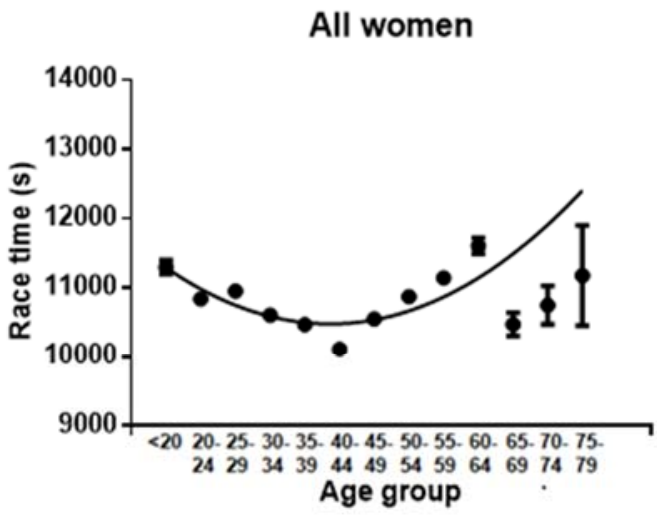

Best women

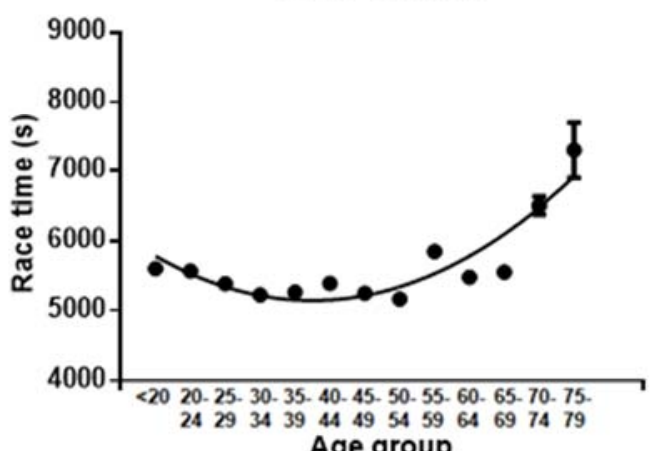

All men

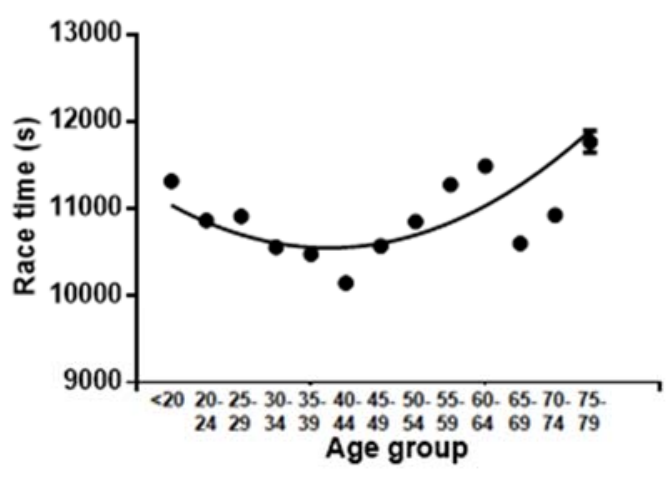

Best men

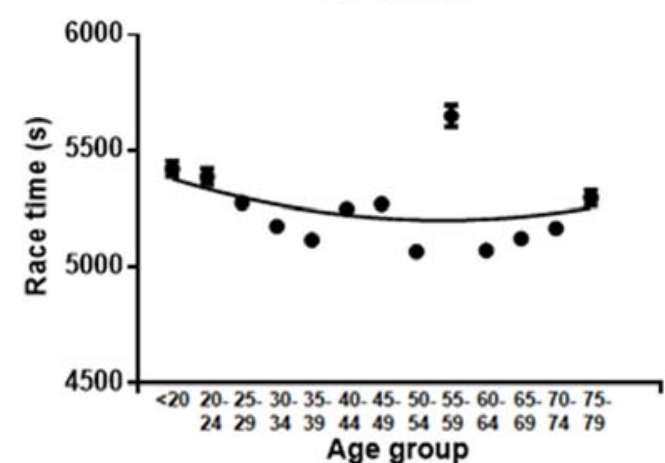

581

582

583

584

585

586

587

588

589

590

591

592

593 
Figure 4
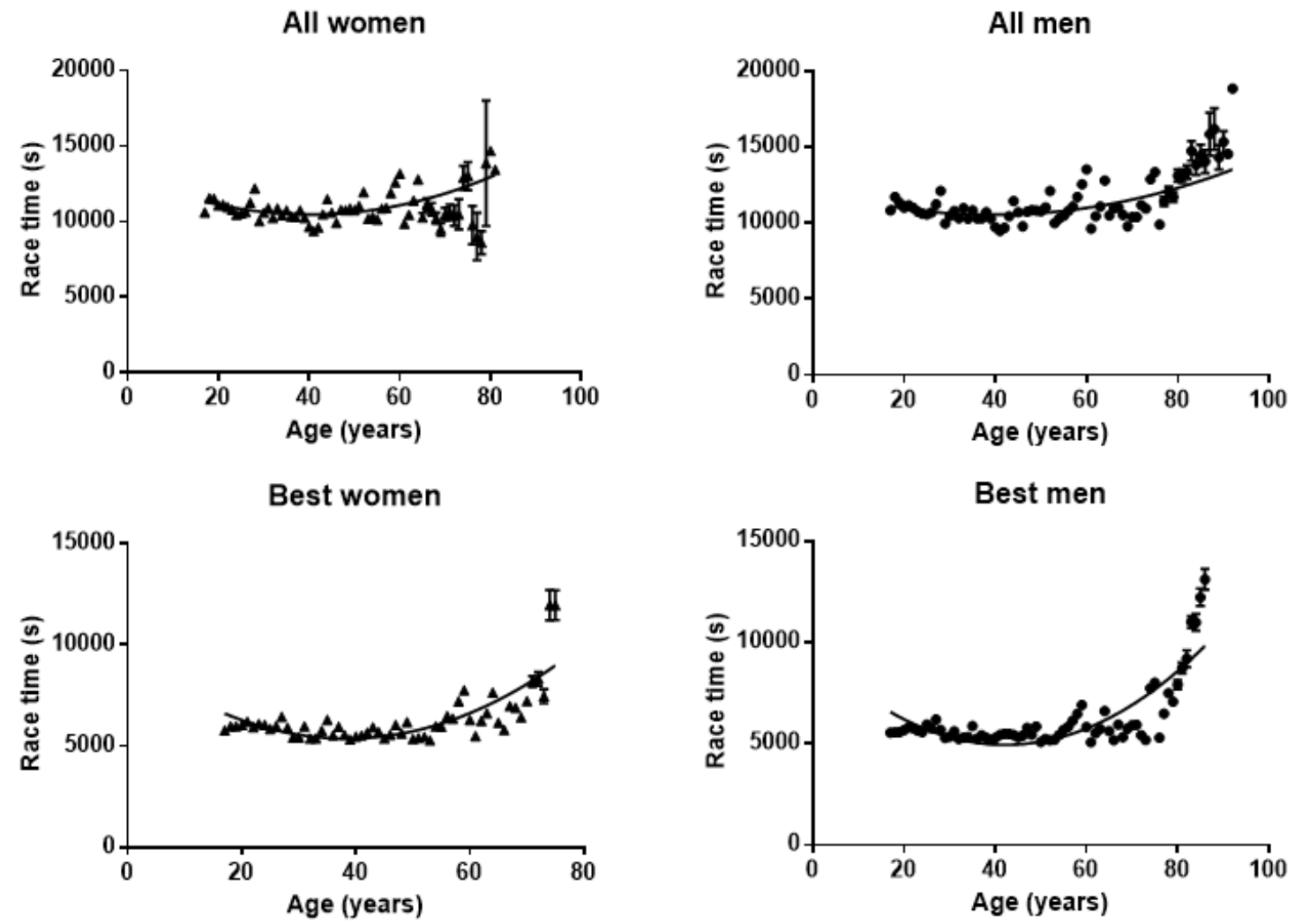

596

597

598

599

600

601

602

603

604

605

606

607

608

609

610 